Technological University Dublin ARROW@TU Dublin

2009-09-01

\title{
An Electrooculogram-based Binary Saccade Sequence Classification (BSSC) Technique for Augmentative Communication and Control
}

\author{
Johnalan Keegan \\ Technological University Dublin, johnalan.keegan@tudublin.ie \\ Ted Burke \\ Technological University Dublin, ted.burke@tudubln.ie \\ James Condron \\ Technological University Dublin, james.condron@tudublin.ie
}

Follow this and additional works at: https://arrow.tudublin.ie/teapotcon

Part of the Biomedical Commons, and the Computer Engineering Commons

\footnotetext{
Recommended Citation

This Article is brought to you for free and open access by the tPOT: People Oriented Technology at ARROW@TU Dublin. It has been accepted for inclusion in Conference Papers by an authorized administrator of ARROW@TU Dublin. For more information, please contact arrow.admin@tudublin.ie, aisling.coyne@tudublin.ie, gerard.connolly@tudublin.ie.
}

Keegan, Johnalan, Burke, Ted, Condron, James: An Electrooculogram-based Binary Saccade Sequence Classification (BSSC) Technique for Augmentative Communication and Control. Engineering in Medicine and Biology Society, 2009. EMBC 2009. Annual International Conference of the IEEE, 3-6 Sept., 2009, Minneapolis, 2009, p.2604-2607. ISSN: 1557-170X ISBN: 978-1-4244-3296-7

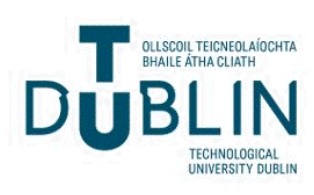




\title{
An Electrooculogram-based Binary Saccade Sequence Classification (BSSC) Technique for Augmentative Communication and Control
}

\author{
Johnalan Keegan, Edward Burke and James Condron
}

\begin{abstract}
In the field of assistive technology, the electrooculogram (EOG) can be used as a channel of communication and the basis of a man-machine interface. For many people with severe motor disabilities, simple actions such as changing the TV channel require assistance. This paper describes a method of detecting saccadic eye movements and the use of a saccade sequence classification algorithm to facilitate communication and control. Saccades are fast eye movements that occurs when a person's gaze jumps from one fixation point to another. The classification is based on pre-defined sequences of saccades, guided by a static visual template (e.g. a page or poster).

The template, consisting of a table of symbols each having a clearly identifiable fixation point, is situated within view of the user. To execute a particular command, the user moves his or her gaze through a pre-defined path of eye movements. This results in a well-formed sequence of saccades which are translated into a command if a match is found in a library of predefined sequences.

A coordinate transformation algorithm is applied to each candidate sequence of recorded saccades to mitigate the effect of changes in the user's position and orientation relative to the visual template. Upon recognition of a saccade sequence from the library, its associated command is executed.

A preliminary experiment in which two subjects were instructed to perform a series of command sequences consisting of 8 different commands are presented in the final sections. The system is also shown to be extensible to facilitate convenient text entry via an alphabetic visual template.
\end{abstract}

\section{INTRODUCTION}

Normal eye movements can be classified into 5 different categories [1], one of which is saccadic movement. Saccades are rapid angular displacements of the eyes that abruptly move them to a new point of fixation. For example, when a person reads a page of text his or her eyes move in a series of 'jolts' rather than smoothly across the page. Saccades can pose problems for systems which attempt to track smooth eye movements for use as a method of control. Our system attempts to take advantage of this type of eye movement by recognising saccade patterns generated by specific eye gestures.

The electrooculogram (EOG) is a biopotential signal recorded from the skin in the vicinity of the eyes. A standing electrical potential exists between the positively charged cornea and the negatively charged retina [1] at the back of the eyeball. This gives rise to an electric field in the vicinity of the eye which moves with the eye. Conventionally, the EOG is recorded in two bipolar leads simultaneously. The first records the voltage between electrodes positioned

J. Keegan, T. Burke and J. Condron are with the School of Electrical Engineering Systems, Dublin Institute of Technology, Kevin St., Dublin 8, Ireland. johnalan.keegan@dit.ie, ted.burke@dit.ie, james.condron@dit.ie

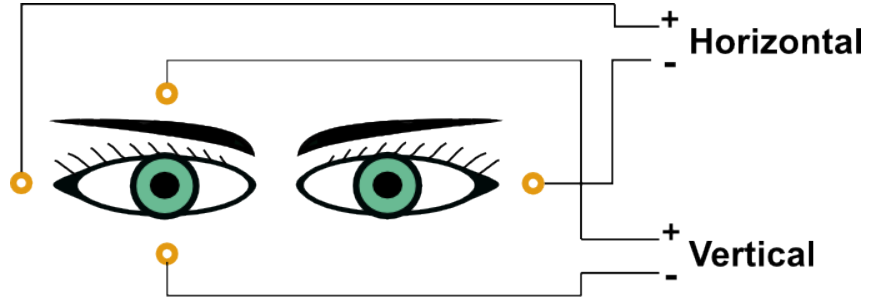

Fig. 1. Typical EOG electrode placements for Horizontal and Vertical channels

near the outer canthi of each eye, which changes when the eyes rotate horizontally. The second records the voltage between electrodes positioned above and below one of the eyes, which changes when the eyes rotate vertically. From the two recorded voltage signals, the movement of the eyes can be inferred approximately, subject to certain limitations. The conventional placement of EOG electrodes for horizontal and vertical channels is illustrated in Fig. 1.

Because of its relatively large amplitude (compared to other biopotentials) and the intuitive nature of the relationship between its two channels and angular displacement of the eyes in the horizontal and vertical directions, the EOG may seem like an ideal candidate for a gaze-tracking system. However, there are certain problems associated with using it in this way. For example, straightforward mapping of the horizontal and vertical EOG channels onto the $\mathrm{X}$ and $\mathrm{Y}$ coordinates of the mouse pointer on a PC requires the use of dc coupled biopotential amplifiers, which can present challenges. In particular, it is difficult to avoid drift in the input voltage offset of each channel (e.g. due to changing electrode voltage offsets), which may necessitate frequent recalibration of such systems during use.

Previous attempts to harness the EOG as a control signal, such as an interface to a computer mouse [2], [3], [4], [5], for text input [6], [7] and for control [8], [9] have produced some promising results but have suffered from common problems such as drift and/or restrictions on head movement.

Burke [10] previously proposed the use of a static target board to guide a subject's saccadic eye movements so that they could be used to control a musical synthesizer during a performance. Here, building on this principle, we describe a novel paradigm for EOG-based communication through recognition of predefined saccade sequences. A user views a target board with a collection of labelled target points. By directing his or her gaze at each of a predefined sequence of points in order, the user executes a command.

To date, our experiments have focused on environmental 
control applications. However, the same principle could be applied to text-entry using an alphanumeric command board.

\section{METHOD}

Each time a new EOG sample is recorded, the following actions are taken:

- Perform saccade detection on the horizontal channel.

- If a saccade is detected, record it as a horizontal and vertical offset and add it to a list of recent saccades.

- Carry out pattern recognition on the sequence of the last $l$ saccades and check for a matching command sequence in the command library.

1) The Command Board: Each command sequence is represented by a unique set of transitions in space from one point to another in a certain order. These command sequences were especially chosen so as to produce a binary output created by the up and down transitions crossing the $\mathrm{x}$-axis in the detected EOG. The user first focuses on the start point and shifts his or her eyes to the first point in the command sequence. Once there, he or she moves to the next point and so on in a rhythmical fashion. The final saccade in each sequence brings the fixation point back to the starting position $(0,0)$.

2) Saccade Detection: The saccade detection algorithm uses the horizontal EOG channel only. The method is to slide a 3-paned window (illustrated in Fig. 2) along the signal looking for times at which

- The mean absolute deviations of the signal in the first (earliest) and third (latest) panes is below a threshold, i.e. the eyes remained fixated during each of these panes.

- The difference between the mean levels in the first and third panes exceeds a threshold, i.e. the position of the eyes must have changed between the first and third panes.

- The mean signal level in the center pane is close to the average of the mean levels in the left and right panes, i.e. the saccade which occurred in the second pane is close to the centre of that pane.

The following expressions define $\bar{x}_{n}$ and $\bar{y}_{n}$, the unweighted prior moving averages of $x$ and $y$ respectively over $N$ samples.

$$
\begin{aligned}
& \bar{x}_{n}=\frac{1}{N} \sum_{i=n-N+1}^{n} x_{i} \\
& \bar{y}_{n}=\frac{1}{N} \sum_{i=n-N+1}^{n} y_{i}
\end{aligned}
$$

Represented as a mean vector,

$$
\overline{\boldsymbol{v}}_{n}=\left[\begin{array}{l}
\bar{x}_{n} \\
\bar{y}_{n}
\end{array}\right]
$$

Furthermore, $\sigma_{x n}$ is the moving mean absolute deviation of $x$ over the same $N$ samples.

$$
\sigma_{x n}=\frac{1}{N} \sum_{i=n-N+1}^{n}\left|x_{i}-\bar{x}_{n}\right|
$$

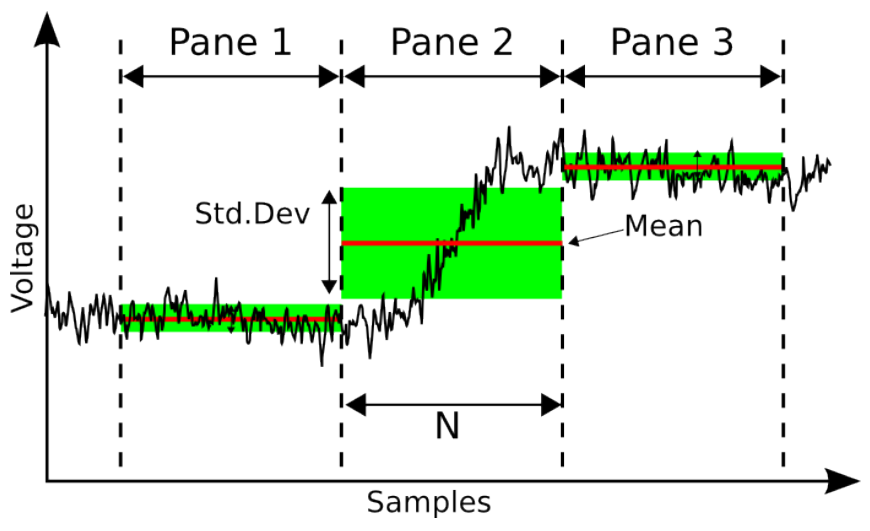

Fig. 2. 3-paned window for saccade detection

Therefore a saccade is recognised at time $n$ if four criteria are satisfied. The first three are

$$
\begin{gathered}
\left|\sigma_{x n-N}\right|>k_{1} \max \left(\left|\sigma_{x n}\right|,\left|\sigma_{x n-2 N}\right|\right) \\
\left|\bar{x}_{n}-\bar{x}_{n-2 N}\right|>k_{2} \max \left(\left|\sigma_{x n}\right|,\left|\sigma_{x n-2 N}\right|\right) \\
\left|\bar{x}_{n}-\bar{x}_{n-N}\right|<\left|\bar{x}_{n-N}-\bar{x}_{n-2 N}\right|
\end{gathered}
$$

The fourth criterion is that a saccade has not been detected for at least $2 N$ samples prior to the current sample. The purpose of this criterion is to prevent multiple detections of a single saccade. $N$ is chosen so as to be large enough to incorporate the largest saccade in the recorded signals.

\section{A. Recording saccades}

For the $m^{t h}$ recognised saccade, detected at sample time $n$, the system records the time of saccade occurrence, $t_{m}$, and the EOG vector offset associated with the saccade, $\boldsymbol{s}_{m}$, which are defined as

$$
t_{m}=n-\frac{3 N}{2}
$$

which corresponds to the middle of the centre pane in Fig. 2, and

$$
\boldsymbol{s}_{m}=\left[\begin{array}{l}
s_{x m} \\
s_{y m}
\end{array}\right]=\overline{\boldsymbol{v}}_{n}-\overline{\boldsymbol{v}}_{n-2 N}
$$

The list of detected saccades is $S$.

$$
S=\left\{s_{0}, s_{1}, s_{2}, \ldots, s_{m}\right\}
$$

For convenience, $S_{l}$ is defined as the subsequence of $S$ made up of its last $l$ saccades.

$$
S_{l}=\left\{s_{m-l+1}, s_{m-l+2}, \ldots, s_{m}\right\}
$$

\section{B. Saccade sequence recognition}

Upon detection of the $m^{\text {th }}$ saccade, a potential match is sought in the library of candidate sequences, $C$. Each element in the command library is a sequence of allowable binary represented commands.

$$
C=\left\{C_{1}, C_{2}, C_{3}, \ldots, C_{L}\right\}
$$

Note that the $L$ candidate sequences in $C$ are not necessarily all of the same length. A recorded saccade sequence $S_{l}$ 
matches a candidate sequence $C_{a}$ of length $l$ if the properties of the sequence passes a number of classification rules.

\section{Pattern Classification}

1) Initial Criteria: Once a saccade is detected in the horizontal channel, the offset values for both channels are stored in their respective memory buffers. $S_{l}$ is then checked against the following criteria:

- the amount of time between the initial saccade and the final saccade is within an allowable limit. That is:

$$
t_{m}-t_{m-l}<t_{\max }
$$

- the sum of the offsets in $S_{l}$ evaluates to a point which is within a certain threshold level of the origin in Euclidian space. That is:

$$
\sum_{i=m-l}^{m} s_{i}<t h_{\max }
$$

2) Transformations: Since each valid command sequence ends with the user's gaze returning to the starting point, the sum of all saccade offset vectors in $S_{l}$ (for a valid sequence) should be close to zero. In fact, DC drift will cause a small error offset to accumulate during the sequence of saccades. As an approximate correction of this drift, the total error offset can be divided by the number of saccades to obtain an estimated per-saccade drift correction offset. The correction is applied to each saccade offset vector, $\boldsymbol{s}_{i}$, to obtain a corrected saccade offset vector, $\boldsymbol{s}_{i}^{\prime}$, as follows.

$$
\boldsymbol{s}_{i}^{\prime}=\boldsymbol{s}_{i}+\frac{\sum_{j=m-l+1}^{m} \boldsymbol{s}_{j}}{l}
$$

Using these new offset values, each is rotated until the last saccade is aligned with the $\mathrm{x}$-axis (as in Fig. 3), using the rotation matrix, $R$, where

$$
R=\left[\begin{array}{cc}
\cos \theta & -\sin \theta \\
\sin \theta & \cos \theta
\end{array}\right]
$$

3) Final feature check: The final test to see if the candidate sequence is valid is to check that all horizontal saccade offsets, $s_{x i}^{\prime}$, except for $s_{x m}^{\prime}$ (the last horizontal offset of the final saccade) are positive. This corresponds to the gaze always moving to the right throughout the sequence until the final saccade when it jumps back to the starting point.

$$
s_{i}^{\prime}>0 \quad, \quad m-l<i<m
$$

Each saccade finishing point in a verified sequence is translated into one bit in the corresponding binary command. The $i^{t h}$ saccade in a sequence of length $l$ is interpreted as a 1 if it finishes above the $\mathrm{x}$-axis and a 0 if it finishes below. In other words,

$$
\sum_{j=m-l+1}^{m-l+i} s_{y j}^{\prime} \geq 0 \Longrightarrow \text { Bit }=1
$$

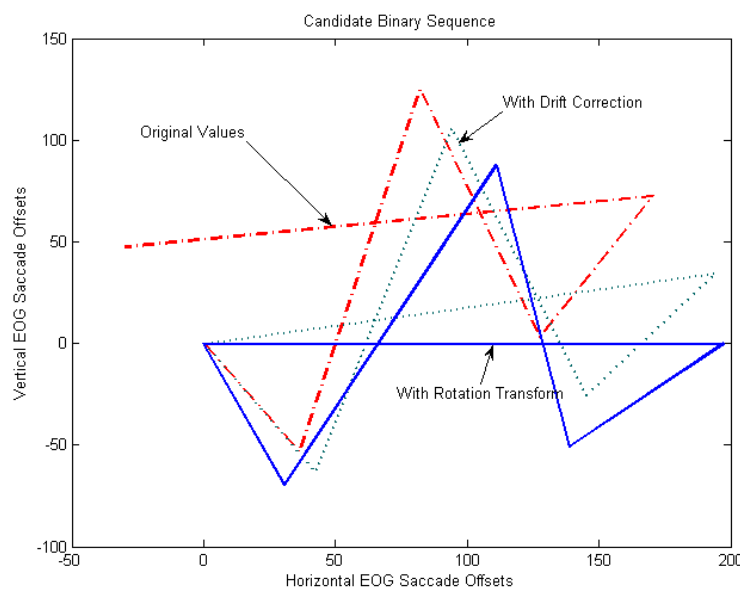

Fig. 3. Transformation of a candidate binary sequence

$$
\sum_{j=m-l+1}^{m-l+i} s_{y j}^{\prime}<0 \Longrightarrow B i t=0
$$

Once the binary translation is complete for each bit in the saccade sequence, the command associated with the binary representation is executed. This could be either a device command or an alphanumeric character for a text entry application. If the candidate sequence does not pass, the oldest saccade is deleted from the buffer and the classification process is repeated once a new saccade is detected.

\section{EXPERIMENTATION}

To test the theory, an experiment was carried out in which 2 subjects aged 25 and 27 used the target board shown in Fig. 4 to perform a selection of predefined saccade sequences. Each participant was seated $150 \mathrm{~cm}$ normal to the target board, situated directly in his or her field of vision. On instruction, they were asked to perform one of the displayed sequences while their horizontal and vertical EOG were recorded.

\section{A. Equipment}

All electrooculogram recording was carried out using a BioSemi ActiveTwo biopotential recording system. The sampling frequency used was $2048 \mathrm{~Hz}$. Situated on a table in front of each participant were two push buttons which were connected to the trigger inputs of the BioSemi system. One button was used to indicate the beginning of a command sequence, while the other was used to indicate that one had been completed. It should be noted that the buttons were used simply to annotate the recorded data and do not play a part in the proposed method of communication. The target board consisted of 8 command sequences printed out on A4 sheets of paper and arranged around a center image, as seen in Fig. 4.

\section{B. Procedure}

Each participant was instructed to perform one of the 8 command sequences labeled $\mathrm{A}-\mathrm{H}$ chosen in a predetermined 


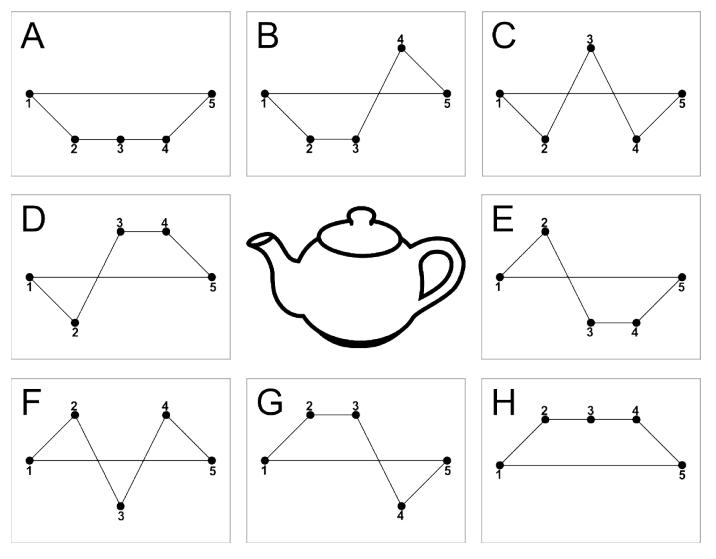

Fig. 4. Command sequence stimuli

random order. This was repeated 5 times for each subject. Before performing the task the subject was instructed to press the left push-button to indicate the beginning of a command sequence, followed by the right push-button upon each sequence completion. He or she was also instructed to avoid blinking or making any head movements while performing a sequence.

\section{RESUlts}

The results of the experiment explained above are presented in Tables I and II. Unrecognised/Missed commands are sequences performed by a test subject which were not recognised as a valid sequence. Incorrect commands are those which were classified as a sequence other than the one performed. In such cases one or more of the fixations appeared on the wrong side of the horizontal after the transformations. Table I shows that the algorithm is capable of yielding an overall accuracy of $93.75 \%$. It can be inferred from Table II that the classification rate is not reliant on any particular saccade sequence.

\section{CONCLUSIONS AND FUTURE WORKS}

\section{A. Conclusions}

The results achieved have shown that the paradigm presented constitutes a practical method of communication and/or control. Although the experiments conducted in these trials used 3-bit binary sequences, allowing a total of 8 different commands, the system is easily extendable to higher resolutions, thus increasing the amount of possible command sequences.

The results have highlighted a large degree of accuracy which warrants further investigation for practical use in the real world.

\section{B. Future Works}

A plan to investigate the classification of different types of saccade patterns is ongoing. The current binary based system relies mainly on the horizontal channel for the detection of saccades. The system also requires two extra saccades for each $n$ bit binary sequence, (i.e. one back to the horizontal plane and another back to the origin). Incorperating
TABLE I

RESULTS OF SACCADE SEQUENCE CLASSIFICATION

\begin{tabular}{lllll}
\hline Subject & Correct & Incorrect & Unrecognised & Accuracy \\
\hline 1 & 38 & 1 & 1 & $95 \%$ \\
2 & 37 & 0 & 3 & $92.5 \%$ \\
\hline \hline Total: & 75 & 1 & 4 & $93.75 \%$ \\
\hline
\end{tabular}

TABLE II

INDIVIDUAL COMMAND ACCURACY

\begin{tabular}{lllll}
\hline Command & Correct & Incorrect & Missed & Accuracy \\
\hline A & 10 & 0 & 0 & $100 \%$ \\
B & 8 & 1 & 1 & $80 \%$ \\
C & 9 & 0 & 1 & $90 \%$ \\
D & 10 & 0 & 0 & $100 \%$ \\
E & 9 & 0 & 1 & $90 \%$ \\
F & 9 & 0 & 1 & $90 \%$ \\
G & 10 & 0 & 0 & $100 \%$ \\
H & 10 & 0 & 0 & $100 \%$ \\
\hline
\end{tabular}

the vertical channel in the saccade detection may enable more accurate classification and allow non-binary patterns of saccade sequences to be classifiable.

\section{REFERENCES}

[1] J. D. Bronzino, The Biomedical Engineering Handbook, Third Edition - 3 Volume Set, 3rd ed. CRC, Apr. 2006.

[2] B. Estrany, P. Fuster, A. Garcia, and Y. Luo, "Accurate interaction with computer by eye movement tracking," in Intelligent Environments, 2008 IET 4th International Conference on, 2008, pp. 1-7.

[3] G. Norris and E. Wilson, "The eye mouse, an eye communication device," in Bioengineering Conference, 1997., Proceedings of the IEEE 1997 23rd Northeast, 1997, pp. 66-67.

[4] H. Manabe and M. Fukumoto, "Full-time wearable headphonetype gaze detector," in CHI '06 extended abstracts on Human factors in computing systems. Montral, Qubec, Canada: ACM, 2006, pp. 1073-1078. [Online]. Available: http://portal.acm.org/citation.cfm?id=1125451.1125655

[5] S. Kwon and H. Kim, "EOG-based glasses-type wireless mouse for the disabled," in [Engineering in Medicine and Biology, 1999. 21st Annual Conf. and the 1999 Annual Fall Meeting of the Biomedical Engineering Soc.] BMES/EMBS Conference, 1999. Proceedings of the First Joint, vol. 1, 1999, p. 592 vol.1.

[6] A. R. Kherlopian, J. P. Gerrein, M. Yue, K. E. Kim, M. Sukumaran, and P. Sajda, "Electrooculogram based system for computer control using a multiple feature classification model," in Engineering in Medicine and Biology Society, 2006. EMBS '06. 28th Annual International Conference of the IEEE, 2006, pp. 1295-1298.

[7] K. Yamagishi, J. Hori, and M. Miyakawa, "Development of EOGBased communication system controlled by Eight-Directional eye movements," in Engineering in Medicine and Biology Society, 2006. EMBS '06. 28th Annual International Conference of the IEEE, 2006, pp. 2574-2577.

[8] R. Barea, L. Boquete, M. Mazo, and E. Lopez, "System for assisted mobility using eye movements based on electrooculography," Neural Systems and Rehabilitation Engineering, IEEE Transactions on, vol. 10, no. 4, pp. 209-218, 2002.

[9] S. Venkataramanan, P. Prabhat, S. Choudhury, H. Nemade, and J. Sahambi, "Biomedical instrumentation based on electrooculogram (EOG) signal processing and application to a hospital alarm system," in Intelligent Sensing and Information Processing, 2005. Proceedings of 2005 International Conference on, 2005, pp. 535-540.

[10] E. Burke, "Accessible human-computer interfaces for communication and creative expression," PhD in Biomedical Engineering, Faculty of Engineering and Architecture, University College Dublin, 2005. 